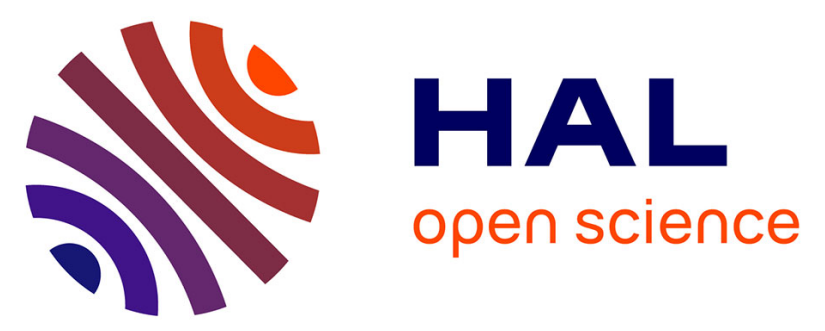

\title{
ETUDE DE L'EVOLUTION STRUCTURALE D'UN VERRE METALLIQUE Fe83B14Si1,5Cl,5 PAR MESURE DU FROTTEMENT INTERIEUR ET DU MODULE DE YOUNG
}

F. Guyot, F. Fouquet, C. Mai, J. Perez

\section{To cite this version:}

F. Guyot, F. Fouquet, C. Mai, J. Perez. ETUDE DE L'EVOLUTION STRUCTURALE D'UN VERRE METALLIQUE Fe83B14Si1,5Cl,5 PAR MESURE DU FROTTEMENT INTERIEUR ET DU MODULE DE YOUNG. Journal de Physique Colloques, 1982, 43 (C9), pp.C9-595-C9-598. 10.1051/jphyscol:19829118 . jpa-00222423

HAL Id: jpa-00222423 https://hal.science/jpa-00222423

Submitted on 1 Jan 1982

HAL is a multi-disciplinary open access archive for the deposit and dissemination of scientific research documents, whether they are published or not. The documents may come from teaching and research institutions in France or abroad, or from public or private research centers.
L'archive ouverte pluridisciplinaire HAL, est destinée au dépôt et à la diffusion de documents scientifiques de niveau recherche, publiés ou non, émanant des établissements d'enseignement et de recherche français ou étrangers, des laboratoires publics ou privés. 
JOURNAL DE PHYSIQUE

Colloque C9, supplément au n¹2, Tome 43, décembre 1982

page $\mathrm{C} 9-595$

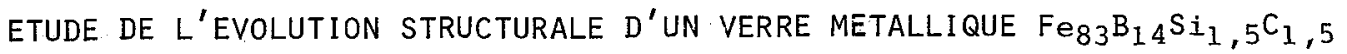
PAR MESURE DU FROTTEMENT INTERIEUR ET DU MODULE DE YOUNG

\author{
F. Guyot, F. Fouquet, C. Mai et J. Perez \\ Groupe d'Etudes de Métallungie Physique et de Physique des Matéxiaux (LA 341) \\ I.N.S.A. de Lyon, Bât 502, 69621 Vilizeurbanne Cedex, France
}

Résumē. - L'influence de la relaxation structurale et du début de la cristallisation sur l'effet $\Delta E$ et $l^{\prime}$ amortissement magnétomécanique a été étudiée dans un verre métallique ferromagnétique $\mathrm{Fe}_{83} \mathrm{~B}_{14} \mathrm{Si}_{1,5} \mathrm{C}_{1,5}$.

Abstract. - The influence of structural relaxation and of the onset of crystallization on $\Delta E$ effect and magnetomechanical damping has been studied in a ferromagnetic metallic glass $\mathrm{Fe}_{83} \mathrm{~B}_{14} \mathrm{~S}_{1.5} \mathrm{C}_{1.5}$.

Introduction. - L'application des techniques de mesure de module élastique et de frottement intérieur au cas des verres métalliques, et en particulier à l'étude de leur évolution structurale, n'a donné lieu, à ce jour, qu'à un nombre de travaux relativement restreint, parmi lesquels on peut citer ceux de SOSHIRODA et al. [1], BERRY [2], KURSOMOVIC et al [3], KÜNZI et a1 [4] et KISS et a1 [5].

Des mesures de pouvoir thermoêlectrique, ainsi que des expériences de diffraction et de diffusion aux petits angles de rayons $X[6]$, nous ont permis de mettre en évidence plusieurs étapes dans 1 'évolution structurale d'un verre métallique de composition Fe83 $\mathrm{B}_{14} \mathrm{Si}_{1,5} \mathrm{C}_{1}, 5$ fábriqué par SAINT-GOBAIN RECHERCHES : (i) en phase vitreuse, un premier stade de mise en ordre topologique, puis un second stade de mise en ordre chimique (ii) lors de la dévitrification, i'apparition de cristallites de solution solide $\mathrm{Fe}-\alpha+\mathrm{Si}$, puis la cristallisation de Ta phase amorphe restante en une phase $\mathrm{Fe}_{2} \mathrm{~B}$ et une phase métastable du type ( $\left.\mathrm{Fe}, \mathrm{Si}\right)_{3}(\mathrm{~B}, \mathrm{C})$ qui se décompose par la suite. Une série d'isothermes nous ont permis de tracer les courbes T.T.T. de T'évolution structurale de cet alliage vitreux [7].

Les mesures de module élastique et de frottement intérieur peuvent mettre en évidence la mobilité atomique dans le matériau ainsi qu'un éventuel couplage magnéto-élastique. L'objectif de ce travail est donc d'obtenir, en liaison avec les travaux antérieurs, une meilleure connaissance du matériau, du point de vue des propriétés magnétiques (magnétoélasticité, tempērature de Curie), et de l'évolution structurale (relaxation structurale et cristallisation).

Méthode expërimentale. - Dans la technique de la "lame vibrante" utilisée, l'échantilTon est encastré à une extrémité et libre à 1'autre. L'excitation et la détection de la vibration se font par voie électrostatique. Le système fonctionnant en boucle fermée, l'êprouvette vibre sur un mode propre avec une amplitude de vibration qui est maintenue constante (environ $2 \cdot 10^{-6}$ ). La fréquence $f$ est alors proportionnelle à $\sqrt{E}$, E étant le module élastique. Pour nos échantillons à 1 'état brut, dont les dimensions sont $0,035 \times 1,1 \times 12 \mathrm{~mm}^{3}$, la fréquence est de 1 'ordre de $150 \mathrm{~Hz}$ sur $7 \mathrm{e}$ mode fondamental. Le frottement intérieur est obtenu à partir du décrément logarithmique mesuré en décroissance libre après coupure de l'excitation. Ces mesures sont rêalisables entre 80 et $700 \mathrm{~K}$, sous un vide de $10^{-3}$ Torr.

Rēsultats expérimentaux. - La figure 1 représente le comportement d'un échantilion brut d'êlaboration lors d'une montée en temperature et du refroidissement consécutif. Le module de Young ne présente que de faibles variations jusqu'à $660 \mathrm{~K}$, température à partir de laquelle il augmente rapidement sous $7^{\prime}$ 'effet de $l^{\prime}$ apparition des premiers cristallites de Fe- $\alpha$. Il est à noter que la seule amorce de la cristallisation suffit à provoquer un accroissement du module de $30 \%$ à la tempërature ambiante. Le frottement intérieur présente une augmentation rapide à partir de $500 \mathrm{~K}$ environ, avec vers $670 \mathrm{~K}$ un maximum dû à la cristaltisation. 


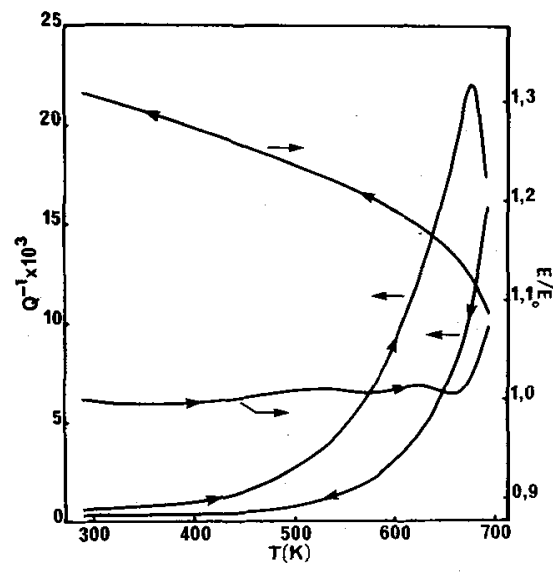

Figure l: Variations du module de Young et du frottement intérieur en fonction de la temperature, a partir de l'êtat brut d'elaboration. E est rapporté à sa valeur $E_{0}$ a 1 'état brut de trempe et à

la température ambiante. Vitesse de chauffage: $1 \mathrm{~K} / \mathrm{min}$.

à $300 \cdot K$. C'est 1 a combinaison de ces 1 'allure des courbes de la figure 1 .

Les courbes 4 de 1 a figure 2 mettant en évidence une augmentation continue de 1 'effet $\Delta \mathrm{E}$ lorsque la température diminue depuis Tc jusqu'à la température ambiante, il nous a semblé intéressant de connaître ce qui se passe à plus basse température. La figure 3 représente le comportement du frottement intérieur et du module de Young d'êchantilions dans différents ètats structuraux. On constate que : (i) après recuit à $573 \mathrm{~K}, 1$ 'ëffet $\Delta E$ et 1 'amortissement magnétomécanique continuent d'augmenter lorsqu'on refroidit jusqu'a la température de l'azote liquide (courbes 2);

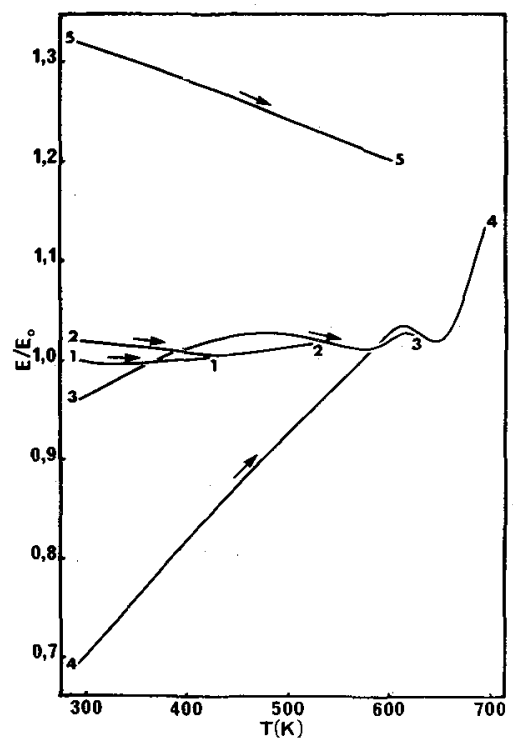

$-\mathbf{a}-$

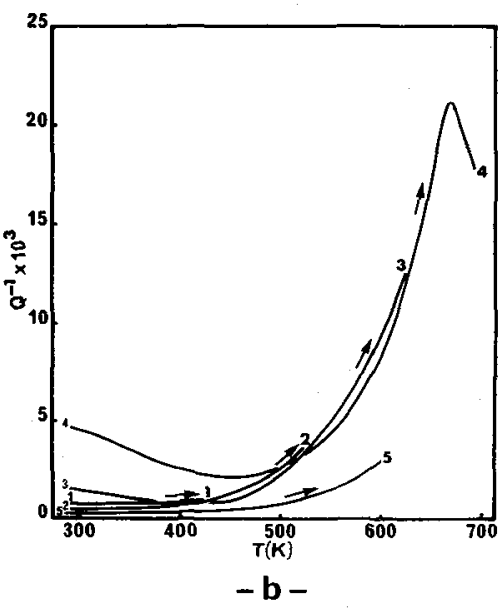

Figure 2: Varlations du module de young (a), et du frottement intérieur (b) en fonction de la température lors de montées successives, suivies d'un refroidissement rapide, jusqu'a: $423 \mathrm{~K}$ (courbes 1) $523 \mathrm{~K}$ (courbes 2), 623 K (courbes 3). $693 \mathrm{~K}$ (courbes 4 ) et $600 \mathrm{~K}$ (courbes 5 ). 
(ii) 1a présence de premiers cristallites de Fe- $\alpha$ provoque une disparition progressive del'effet $\triangle E$ avec apparition d'un maximum de frottement intérieur et d'un minimum de module de Young quị se déplacent vers les plus hautes températures au fur et à mesure que la cristallisation progresse (courbes 3-4-5).

Discussion. - L'existence d'un effet $\Delta E$ dans des verres du type Fe-P-C a déjà été signalée et étudiée par BERRY et PRITCHET $[9,10]$. Toutefois dans notre a11 iage, 1 'amplitude de 7 'effet atteint des valeurs plus importantes.

A l'êtat brut d'élaboration l'effet magnétoèlastique est faible, car les contraintes internes sont très importantes. Lors d'un recuit sans cristallisation, la relaxation structurale a pour effet, d'une part un relachement des contraintes internes rendant plus facile le mouvement des parois et la rotation des domaines, et d'autre part une modification de la structure magnétique étroitement liée à l'êtat de contraintes dans le matériau. L'absence d'énergie d'anisotropie d'origine magnétocristalline favorise encore le couplage et permet à l'effet $\Delta E$ de se développer quand la température décroit.

Lorsque la cristallisation s'amorce, la diminution progressive observée de l'effet $\triangle E$ s'explique par 1'apparition de petits cristallites de Fe- $\alpha$ qui vraisemblablement provoquent à basse température un ancrage des paroiss et 7 a création de "centres" de contraintes et donc d'une anisotropie d'origine magnétostrictive. L'effet $\Delta E$ pourrait réapparaître lorsqu' on chauffe depuis la température de l'azote liquide grâce au désancrage thermiquement activé des parois de domaines : celà conduirait alors à un maximum de frottement intérieur (figure $3-b$, courbes 3 et 4 ).

$L$ 'effet magnétoélastique et 1 'amortissement magnétomécanique d' un matériau ferromagnétique font intervenir différents mécanismes [11]: (i) un processus de relaxation rësultant des courants de Foucault à 1 'échelle macroscopique et microscopique, et indépendant de 1 'amplitude de la déformation $\varepsilon$; ( $i i)$ un processus d'hystêrésis magnétomécanique, indépendant de la fréquence mais qui dépend fortement de $\varepsilon$.

Dans l'état désaimanté, la composante macroscopique du processus de relaxation, qui concerne 1 'aimantation globale du matériau, est nulle. Dans ce cas, quand $\varepsilon$ tend vers zêro, la composante hystêrétique s'annulle, et seule intervient la contribution relaxationnelle par microcourants de Foucault qui tient compte des variations locales de la configuration de domaines. Lorsque $\varepsilon$ augmente tout en restant faible, le phénohềne d'hystérésis apparaît, et l'effet $\Delta E$ ainsi que 1 'amortissement doivent augmenter linéairement avec $\varepsilon$. C'est ce qu'on observe sur. la figure 4 . Nos résultats ayant étê obtenus pour une valeur de $\varepsilon_{\max }$ de $210^{-6}$ environ, la contribution hystérétique
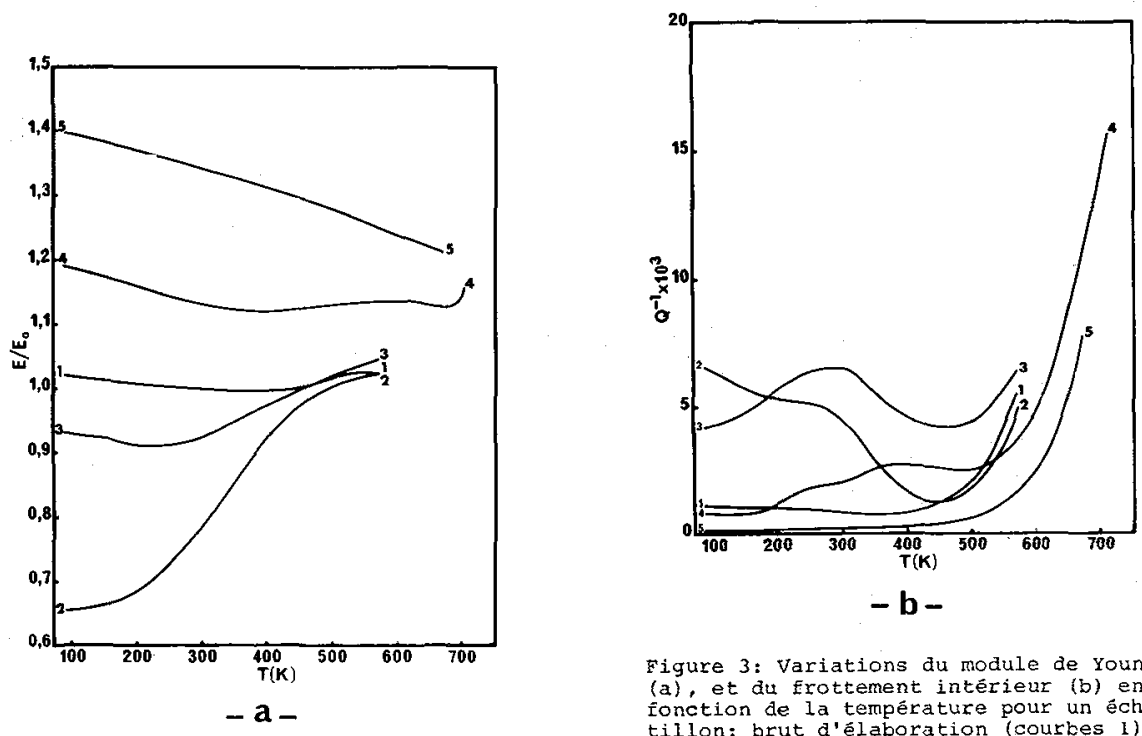

Figure 3: Variations du module de young (a), et du Erottement interieur (b) en Ionction de la température pour un échantillon: brut d'élaboration (courbes 1 ), recuit $145 \mathrm{~min}$. a $573 \mathrm{~K}$ (courbes 2), et recuit $145 \mathrm{~min}$. a $573 \mathrm{~K}$ (courbes 2 ), et 3 ), $680 \mathrm{~K}$ (courbes 4) et $700 \mathrm{~K}$ (courbes 5). 


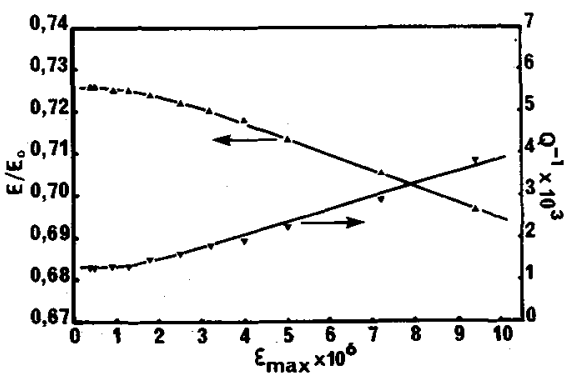

Figure 4: Variations du module de Young et du frottement intérieur en fonction de $\varepsilon_{\max }$ pour un échantilion ayant subi un recuit de stabilisation a $573 \mathrm{~K}$.

existe donc mais reste faible.

Contrairement à ce qu'à dit BERRY [10], un bref calcul nous a montré que le frottement intérieur à amplitude nulle pouvait être interprété uniquement en termes de relaxation par microcourants de Foucault. Toutefois, une mesure sur le premier harmonique, à $800 \mathrm{~Hz}$ environ, nous a montrê qu'il ne variajt pratiquement pas avec la fréquence. Ceci nous conduit donc à admettre que le frottement intêrieur déterminé à amplitude nulle implique une autre contribution, qui ne dépend pas de la fréquence, et qui constituerait une sorte de fond continu magnétomécanique.

Conclusion. - Les mesures de module de Young et de frottement intérieur nous ont permis sur un verre métallique Fe83 $\mathrm{B}_{14} \mathrm{Si}_{1}, 5, \mathrm{C}_{1,5}$ : de mettre en évidence un effet magnétoélastique et un amortissement magnétómécanique, de déterminer la température de Curie, d'étudier la relaxation structurale et de détecter l'apparition des premiers cristallites dans la matrice vitreuse.

Rēférences

[1] T. SOSHIRODA, M. KOIWA, T. MASUMOTO, J. Non Cryst. Solids, $22(1976), 173$.

[2] B. S. BERRY in "Metall ic Glasses" ASM, 161

[3] A. KURSOMOVIC, M.G. SCOTT, E. GIRT, R.W. CAHN, Scripta Met., 14 (1980), 1303.

[4] K. AGYEMAN, E. ARMBRUSTER, H. U. KUNZI, A. DAS GUPTA, H.J. GÜNTHERODT, J. Physique, Co11. C5 supp1. $n^{\circ} 10$ (1981), 535.

[5] S. KISS, G. POSGAY, I.Z. HARANGOZ0, F.J. KEDVES, J. Physique Col1. C5 suppl.n ${ }^{\circ} 10$ (1981), 529.

[6] D.C. TIAN, F. FOUQUET, F. GUYOT, C. MAI, J. PEREZ, Mat. Sci. Eng. 53 (1982), 179.

[7] F. FOUQUET, F. GUYOT, C. MAI, J. PEREZ, D.C. TIAN, Commùnication au colloque "Verres et amorphes" du Congrès de la SFP - Clermont-Ferrand 29 juin-4 juillet 1981.

[8] A.I. TAUB, F. SPAEPEN, Acta Met. 28 (1980), 1781.

[9] B.S. BERRY, W.C. PRITCHET, AIP Conf. Proc. 34 (1976) 292.

[10] B.S. BERRY, W.C. PRITCHET, J. Appl. Phys. 47 (1976) 3295.

[11] A.S. NOWICK, 3.S. BERRY in "Anelastic Relaxation in Crystalline Solids" Academic Press llew York 1972 chap. 18. 